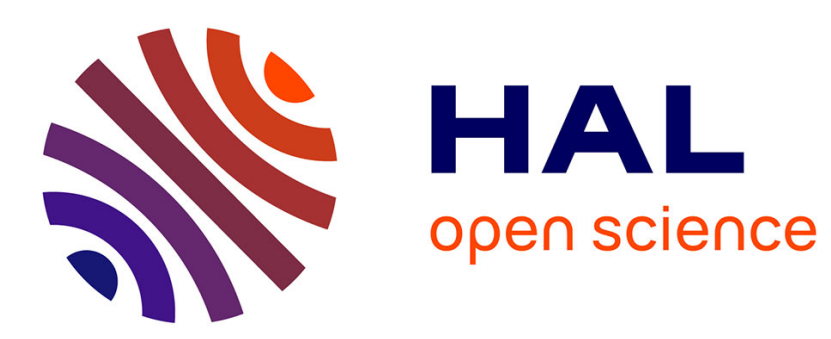

\title{
Observer-based control of discrete-time LPV systems with uncertain parameters
}

\author{
Maurice Heemels, Jamal Daafouz, Gilles Millérioux
}

\section{To cite this version:}

Maurice Heemels, Jamal Daafouz, Gilles Millérioux. Observer-based control of discrete-time LPV systems with uncertain parameters. IEEE Transactions on Automatic Control, 2010, 55 (9), pp.21302135. 10.1109/TAC.2010.2051072 . hal-00529186

\section{HAL Id: hal-00529186 https://hal.science/hal-00529186}

Submitted on 29 Nov 2010

HAL is a multi-disciplinary open access archive for the deposit and dissemination of scientific research documents, whether they are published or not. The documents may come from teaching and research institutions in France or abroad, or from public or private research centers.
L'archive ouverte pluridisciplinaire HAL, est destinée au dépôt et à la diffusion de documents scientifiques de niveau recherche, publiés ou non, émanant des établissements d'enseignement et de recherche français ou étrangers, des laboratoires publics ou privés. 


\title{
Observer-Based Control of Discrete-Time LPV Systems with Uncertain Parameters
}

\author{
W.P.M.H. Heemels, J. Daafouz, G. Millerioux
}

\begin{abstract}
In this paper LMI-based design conditions are presented for observer-based controllers that stabilize discretetime LPV systems in the situation where the parameters are not exactly known, but are only available with a finite accuracy. The presented framework allows to make tradeoffs between the admissible level of parameter uncertainty on the one hand and the transient performance on the other. In addition, the level of parameter uncertainty can be maximized while still guaranteeing closed-loop stability.
\end{abstract}

Index Terms-LPV systems, output feedback and observers, robust control, LMIs, separation principle

\section{INTRODUCTION}

Linear Parameter-Varying (LPV) systems and controllers have received considerable attention from the control community in recent years [2-4,6,15-17]. When LPV controllers are implemented in practice two important properties need to be satisfied. First of all, the controller needs to be output-based, as in practice it is rarely the case that the full state variable is available for feedback. Secondly, the controller must be robust with respect to some degree of mismatch between the available and the true parameters as the real parameters are not always known exactly, although this is often assumed in the literature on LPV systems. This paper will address the design of stabilizing controllers for discretetime LPV systems that satisfy these two properties.

In $[2,12]$ the continuous-time version of this problem was considered, but, unfortunately, only conditions in terms of bilinear matrix inequalities (BMIs) were presented. Only recently a solution was given in [8] using convex programming techniques. In the discrete-time case output-based control design for LPV systems for which the measured parameters do not exactly fit the real ones is at present an open problem. In [14] it is shown that an observer that is asymptotically recovering the state when the parameters are exactly measured, is input-to-state stable (ISS) $[10,18]$ with respect to mismatch between the true and the available parameters. However, [14] does not study the observer synthesis nor the output-based stabilization problem. These two important problems will be solved in this paper.

Closely related to LPV systems are switched linear (SL) systems and piecewise affine (PWA) systems, which can

Maurice Heemels (M.Heemelsetue.nl) is with the Hybrid and Networked Systems group, Dept. Mechanical Engineering, Eindhoven University of Technology, The Netherlands. Jamal Daafouz (jamal.daafouz@ensem.inpl-nancy.fr) and Gilles Millerioux (gilles.milleriouxdesstin.uhp-nancy.fr) are with CRAN, UMR CNRS 7039, Nancy University, France. This work was partially supported by the ANR project ArHyCo, ARPEGE, number ANR-2008 SEGI 004 and the European Committee through the EU-FP7 project MOBY-DIC (no. 248858). be perceived as a subclass of LPV systems in which the parameters only take a finite number of values. Observer-based control design for SL systems has been considered in [5] under the assumption of having exact knowledge of the parameter values. In case of unknown parameters, [1] proposes design conditions for observers that include an estimation procedure for the parameters. In [11,19] observers and observer-based controllers were designed for PWA systems based on LMIs. In this case the parameters are also unknown as they depend on the state variable that has to be estimated. However, as for SL and PWA systems the number of parameter values is finite, these results are not applicable to general LPV systems.

This paper provides a solution to the open problem of output-based controller design for discrete-time LPV systems with uncertain parameters. The main contributions are LMIbased conditions for the separate design of state observers and input-to-state stabilizing state feedbacks for discrete-time LPV systems. We prove that the resulting closed-loop system is globally exponentially stable for some level of mismatch between the true parameters and the available ones. The flexibility in our framework allows to make tradeoffs between the level of mismatch and the transient performance of the closed loop in terms of the decay factor. Moreover, the level of parameter uncertainty can be maximized while still guaranteeing closed-loop stability.

\section{NOTATION AND BASIC DEFINITIONS}

$\mathbb{R}, \mathbb{R}_{\geq 0}$, and $\mathbb{N}$ are the field of real numbers, the set of non-negative reals and the set of non-negative integers, respectively. The $i$-th entry of a real vector $x$ is denoted by $x^{i}$ (subscripts are used for denoting discrete-time dependence). We denote by $\|x\|=\sqrt{x^{T} x}$ the Euclidean norm of $x$ in $\mathbb{R}^{n}$, where $M^{T}$ denotes the transpose for a vector or matrix $M$, and by $\|x\|_{\infty}$ its infinity norm given by $\max _{i}\left|x^{i}\right|$. For a sequence $\left\{v_{k}\right\}_{k \in \mathbb{N}}$ with $v_{k} \in \mathbb{R}^{n}$ we denote its supremum norm $\sup _{k \in \mathbb{N}}\left\|v_{k}\right\|$ by $\|v\|_{\infty}$. For a matrix $M \in \mathbb{R}^{n \times m}$ we denote its spectral norm $\sqrt{\lambda_{\max }\left(M^{T} M\right)}$ by $\|M\|$, where $\lambda_{\max }\left(M^{T} M\right)$ denotes the largest eigenvalue of $M^{T} M$. When a matrix $P$ is positive definite (including symmetry), we write $P \succ 0$. If it is positive semi-definite, we use $P \succeq 0$. Similarly, for (semi-)negative definiteness we write $\prec$ and $\preceq$. By $\mathbf{0}$ and 1 we denote the zero and the identity matrix of appropriate dimensions.

A function $\varphi: \mathbb{R}_{+} \rightarrow \mathbb{R}_{+}$belongs to class $\mathcal{K}$ if it is continuous, strictly increasing and $\varphi(0)=0$ and to class $\mathcal{K}_{\infty}$ if additionally $\varphi(s) \rightarrow \infty$ as $s \rightarrow \infty$. A function $\beta: \mathbb{R}_{+} \times \mathbb{R}_{+} \rightarrow \mathbb{R}_{+}$belongs to class $\mathcal{K} \mathcal{L}$ if for each fixed $k \in \mathbb{R}_{+}, \beta(\cdot, k) \in \mathcal{K}$ and for each fixed $s \in \mathbb{R}_{+}, \beta(s, \cdot)$ 
is decreasing and $\lim _{k \rightarrow \infty} \beta(s, k)=0$. Consider now the discrete-time nonlinear systems

$$
x_{k+1}=G\left(x_{k}, \omega_{k}\right),
$$

and

$$
x_{k+1}=G_{v}\left(x_{k}, v_{k}, \omega_{k}\right),
$$

where $x_{k} \in \mathbb{R}^{n}$ is the state, $v_{k} \in \mathbb{R}^{d_{v}}$ is an unknown disturbance input and $\omega_{k} \in \mathbb{R}^{d_{\omega}}$ is an uncertainty parameter at discrete time $k \in \mathbb{N}$. $G: \mathbb{R}^{n} \times \mathbb{R}^{d_{\omega}} \rightarrow \mathbb{R}^{n}$ and $G_{v}: \mathbb{R}^{n} \times \mathbb{R}^{d_{v}} \times \mathbb{R}^{d_{\omega}} \rightarrow \mathbb{R}^{n}$ are arbitrary nonlinear functions. We assume that $\omega_{k} \in \Omega, k \in \mathbb{N}$ for some set $\Omega \subset \mathbb{R}^{d_{\omega}}$.

Definition 1: $[10,18]$ The system (1) with uncertainty set $\Omega$ is called globally asymptotically stable (GAS), if there exists a $\mathcal{K} \mathcal{L}$-function $\beta$ such that, for each $x_{0} \in \mathbb{R}^{n}$ and all $\left\{\omega_{k}\right\}_{k \in \mathbb{N}}$ with $\omega_{k} \in \Omega, k \in \mathbb{N}$, it holds that the corresponding state trajectory satisfies $\left\|x_{k}\right\| \leq \beta\left(\left\|x_{0}\right\|, k\right)$ for all $k \in \mathbb{N}$. If $\beta$ can be taken of the form $\beta(s, k)=d s \lambda^{k}$ for some $d \geq 0$ and $0 \leq \lambda<1$ the system (1) with uncertainty set $\Omega$ is called globally exponentially stable (GES). The system (2) with uncertainty set $\Omega$ is said to be input-to-state stable (ISS) with respect to $v$ if there exist a $\mathcal{K} \mathcal{L}$-function $\beta$ and a $\mathcal{K}$ function $\gamma$ such that, for each $x_{0} \in \mathbb{R}^{n}$, all $\left\{v_{k}\right\}_{k \in \mathbb{N}}$ and all $\left\{\omega_{k}\right\}_{k \in \mathbb{N}}$ with $\omega_{k} \in \Omega, k \in \mathbb{N}$, it holds for all $k \in \mathbb{N}$ that $\left\|x_{k}\right\| \leq \beta\left(\left\|x_{0}\right\|, k\right)+\gamma\left(\|v\|_{\infty}\right)$.

We call $\lambda$ a decay factor for (1) and the function $\gamma$ an ISS gain of (2). Next we state sufficient conditions for ISS using so-called ISS Lyapunov functions. The proofs are omitted for shortness, but can be based on $[10,13]$ by adopting parameterdependent Lyapunov functions.

Theorem 1: Let $d_{1}, d_{2} \in \mathbb{R}_{\geq 0}$, let $a, b, c, \mu \in \mathbb{R}_{>0}$ with $c \leq b$ and let $\alpha_{1}(s):=a s^{\mu}, \alpha_{2}(s):=b s^{\mu}, \alpha_{3}(s):=c s^{\mu}$ and $\sigma \in \mathcal{K}$. Furthermore, let $V: \mathbb{R}^{n} \times \mathbb{R}^{d_{\omega}} \rightarrow \mathbb{R}_{\geq 0}$ be a function such that

$$
\begin{aligned}
\alpha_{1}(\|x\|) \leq V(x, \omega) & \leq \alpha_{2}(\|x\|) \\
V\left(G_{v}\left(x, v, \omega_{1}\right), \omega_{2}\right)-V\left(x, \omega_{1}\right) & \leq-\alpha_{3}(\|x\|)+\sigma(\|v\|)
\end{aligned}
$$

for all $x \in \mathbb{R}^{n}$, all $v \in \mathbb{R}^{d_{v}}$ and $\omega, \omega_{1}, \omega_{2} \in \Omega$. Then system (2) with uncertainty set $\Omega$ is ISS with respect to $v$. In case (3a) and $V\left(G\left(x, \omega_{1}\right), \omega_{2}\right)-V\left(x, \omega_{1}\right) \leq-\alpha_{3}(\|x\|)$ hold for all $x$ and $\omega, \omega_{1}, \omega_{2} \in \Omega$, then system (1) with uncertainty set $\Omega$ is GES with decay factor $1-\frac{c}{b} \in[0,1)$.

A function $V$ that satisfies (3) is called an ISS Lyapunov function.

\section{PROBLEM STATEMENT}

We consider discrete-time linear parameter-varying (LPV) systems given by

$$
\begin{aligned}
x_{k+1} & =A\left(\rho_{k}\right) x_{k}+B u_{k} \\
y_{k} & =C x_{k}+D u_{k}
\end{aligned}
$$

with $x_{k} \in \mathbb{R}^{n}, y_{k} \in \mathbb{R}^{m}$ and $u_{k} \in \mathbb{R}^{r}$ the state, output and control input at discrete time $k \in \mathbb{N}, \rho_{k} \in \mathbb{R}^{L}$ is a timevarying parameter and $A(\rho) \in \mathbb{R}^{n \times n}$ for each $\rho, B \in \mathbb{R}^{n \times r}$, $C \in \mathbb{R}^{m \times n}, D \in \mathbb{R}^{m \times r}$. The parameter $\rho_{k}, k \in \mathbb{N}$ lies in some set $\Theta \subset \mathbb{R}^{L}$ and we assume that $A: \Theta \rightarrow \mathbb{R}^{n \times n}$ can be written in the polytopic form $A(\rho)=\sum_{i=1}^{N} \xi^{i}(\rho) A_{i}$ for certain continuous functions $\xi^{i}: \Theta \rightarrow \mathbb{R}$ and matrices $A_{i} \in \mathbb{R}^{n \times n}, i=1, \ldots, N$. In addition we assume that the mapping $\xi: \Theta \rightarrow \mathbb{R}^{N}$ given by $\xi:=\left(\xi^{1}, \ldots, \xi^{N}\right)^{\top}$ is such that $\xi(\Theta) \subset \mathcal{S}$ with $\mathcal{S}=\left\{\mu \in \mathbb{R}^{N} \mid \mu^{i} \geq 0, i=\right.$ $1, \ldots, N$ and $\left.\sum_{i=1}^{N} \mu^{i}=1\right\}$. Hence, $A(\rho)$ lies for each $\rho \in \Theta$ in the convex hull $\mathbf{C o}\left\{A_{1}, \ldots, A_{N}\right\}$.

In this paper, we focus on the situation where the true (timevarying) parameter $\rho_{k}$ is not available, but only an estimated parameter $\hat{\rho}_{k} \in \Theta$ fulfilling $\left\|\rho_{k}-\hat{\rho}_{k}\right\|_{\infty} \leq \Delta$ is known, where $\Delta$ is some nonnegative constant indicating the uncertainty level.

Problem 1: Design an observer-based controller

$$
\begin{aligned}
\hat{x}_{k+1} & =A\left(\hat{\rho}_{k}\right) \hat{x}_{k}+B u_{k}+L\left(\hat{\rho}_{k}\right)\left(y_{k}-\hat{y}_{k}\right) \\
\hat{y}_{k} & =C \hat{x}_{k}+D u_{k} \\
u_{k} & =K\left(\hat{\rho}_{k}\right) \hat{x}_{k}
\end{aligned}
$$

with $L\left(\hat{\rho}_{k}\right)=\sum_{i=1}^{N} \xi^{i}\left(\hat{\rho}_{k}\right) L_{i}$ and $K\left(\hat{\rho}_{k}\right)=\sum_{i=1}^{N} \xi^{i}\left(\hat{\rho}_{k}\right) K_{i}$ by appropriately choosing the gains $L_{i}$ and $K_{i}, i=1, \ldots, N$ such that the closed-loop system (4)-(5) is GAS when the uncertainty satisfies $\left\|\rho_{k}-\hat{\rho}_{k}\right\|_{\infty} \leq \Delta$ and $\hat{\rho}_{k} \in \Theta$ for all $k \in \mathbb{N}$.

\section{OBSERVER DESIGN}

We first focus on the estimation of the state $x_{k}$ using a polytopic observer of the form

$$
\left\{\begin{array}{l}
\hat{x}_{k+1}=A\left(\hat{\rho}_{k}\right) \hat{x}_{k}+B u_{k}+L\left(\hat{\rho}_{k}\right)\left(y_{k}-\hat{y}_{k}\right) \\
\hat{y}_{k}=C \hat{x}_{k}+D u_{k},
\end{array}\right.
$$

where $\hat{\rho}_{k} \in \Theta$ and possibly $\rho_{k} \neq \hat{\rho}_{k}$. The estimation error $e_{k}:=x_{k}-\hat{x}_{k}$ is governed by

$$
e_{k+1}=\mathcal{A}_{e}\left(\hat{\rho}_{k}\right) e_{k}+v_{k}
$$

with $\mathcal{A}_{e}\left(\rho_{k}\right):=\sum_{i=1}^{N} \xi^{i}\left(\rho_{k}\right) \tilde{A}_{i}$, where $\tilde{A}_{i}=A_{i}-L_{i} C$ and

$$
v_{k}=(\underbrace{A\left(\rho_{k}\right)-A\left(\hat{\rho}_{k}\right)}_{=: \Delta A\left(\rho_{k}, \hat{\rho}_{k}\right)}) x_{k}
$$

Theorem 2: Assume that there exist symmetric matrices $P_{i} \in \mathbb{R}^{n \times n}$, matrices $G_{i} \in \mathbb{R}^{n \times n}, F_{i} \in \mathbb{R}^{n \times m}, i=1, \ldots, N$ and a scalar $\sigma_{e v} \geq 1$ satisfying for all $i, j=1, \ldots, N$ the LMIs

$$
\left[\begin{array}{cccc}
G_{i}^{T}+G_{i}-P_{j} & \mathbf{0} & G_{i} A_{i}-F_{i} C & G_{i} \\
\mathbf{0} & \mathbf{1} & \mathbf{1} & \mathbf{0} \\
A_{i}^{T} G_{i}^{T}-C^{T} F_{i}^{T} & \mathbf{1} & P_{i} & \mathbf{0} \\
G_{i}^{T} & \mathbf{0} & \mathbf{0} & \sigma_{e v} \mathbf{1}
\end{array}\right] \succ \mathbf{0}
$$

then the error dynamics (7) with uncertainty set $\Theta$ for $\hat{\rho}$ and $^{1}$ $L_{i}=G_{i}^{-1} F_{i}$ is ISS with respect to $v$ with ISS gain $\gamma(s)=$ $\sigma_{e v} s, s \in \mathbb{R}_{\geq 0}$. Moreover, $V_{e}\left(e_{k}, \hat{\xi}_{k}\right)=e_{k}^{T}\left(\sum_{i=1}^{N} \hat{\xi}_{k}^{i} P_{i}\right) e_{k}$ is an ISS Lyapunov function that satisfies for all $\xi_{k}, \hat{\xi}_{k+1} \in \mathcal{S}$, $e_{k} \in \mathbb{R}^{n}, v_{k} \in \mathbb{R}^{n}$

$$
\begin{gathered}
V_{e}\left(e_{k+1}, \hat{\xi}_{k+1}\right)-V_{e}\left(e_{k}, \hat{\xi}_{k}\right) \leq-\left\|e_{k}\right\|^{2}+\sigma_{e v}\left\|v_{k}\right\|^{2}, \\
\left\|e_{k}\right\|^{2} \leq V_{e}\left(e_{k}, \hat{\xi}_{k}\right) \leq \sigma_{e v}\left\|e_{k}\right\|^{2} .
\end{gathered}
$$

${ }^{1}$ The LMIs (9) imply that $G_{i}$ is invertible for each $i=1, \ldots, N$ as is shown in the proof. 
Proof: The feasibility of the LMIs (9) for all $i, j=$ $1, \ldots, N$ implies that

$$
\left[\begin{array}{cc}
\mathbf{1} & \mathbf{1} \\
\mathbf{1} & P_{i}
\end{array}\right] \succ \mathbf{0} \text { and }\left[\begin{array}{cc}
G_{i}^{T}+G_{i}-P_{j} & G_{i} \\
G_{i}^{T} & \sigma_{e v} \mathbf{1}
\end{array}\right] \succ \mathbf{0}
$$

are satisfied for all $i, j=1, \ldots, N$. From (11) it follows that $P_{i} \succ \mathbf{1}$ and $G_{i}^{T}+G_{i}-P_{j} \succ \sigma_{e v}^{-1} G_{i} G_{i}^{T} \succeq 0$ for all $i, j=$ $1, \ldots, N$. Since $\left(P_{j}^{-\frac{1}{2}} G_{i}^{T}-P_{j}^{\frac{1}{2}}\right)^{T}\left(P_{j}^{-\frac{1}{2}} G_{i}^{T}-P_{j}^{\frac{1}{2}}\right) \succeq 0$ implies

$$
G_{i} P_{j}^{-1} G_{i}^{T} \succeq G_{i}+G_{i}^{T}-P_{j},
$$

it follows now that $G_{i} P_{j}^{-1} G_{i}^{T} \succeq \sigma_{e v}^{-1} G_{i} G_{i}^{T}$ and thus $P_{j} \preceq$ $\sigma_{e v} \mathbf{1}, j=1, \ldots, N$, because $G_{i}$ is invertible. Invertibility of $G_{i}$ follows from $G_{i}^{T}+G_{i} \succ P_{j}$ as it would imply for $G_{i} x=0$ that $x^{T} P_{j} x \leq 0$ and thus $x=0$. As such, we have (10b) for all $e_{k} \in \mathbb{R}^{n}$ and all $\hat{\xi}_{k} \in \mathcal{S}$.

To prove (10a), note that feasibility of the LMIs (9) gives together with (12) for all $i, j$ the LMI

$$
\left[\begin{array}{cccc}
G_{i} P_{j}^{-1} G_{i}^{T} & \mathbf{0} & G_{i} A_{i}-F_{i} C & G_{i} \\
\mathbf{0} & \mathbf{1} & \mathbf{1} & \mathbf{0} \\
A_{i}^{T} G_{i}^{T}-C^{T} F_{i}^{T} & \mathbf{1} & P_{i} & \mathbf{0} \\
G_{i}^{T} & \mathbf{0} & \mathbf{0} & \sigma_{e v} \mathbf{1}
\end{array}\right] \succ \mathbf{0 .}
$$

This is equivalent for all $i, j$ to

$$
N_{i j} \Psi_{i j} N_{i j}^{T} \succ \mathbf{0} \text { with } N_{i j}=\left[\begin{array}{cccc}
G_{i} P_{j}^{-1} & \mathbf{0} & \mathbf{0} & \mathbf{0} \\
\mathbf{0} & \mathbf{1} & \mathbf{0} & \mathbf{0} \\
\mathbf{0} & \mathbf{0} & \mathbf{1} & \mathbf{0} \\
\mathbf{0} & \mathbf{0} & \mathbf{0} & \mathbf{1}
\end{array}\right]
$$

and

$$
\Psi_{i j}=\left[\begin{array}{cccc}
P_{j} & \mathbf{0} & P_{j}\left(A_{i}-L_{i} C\right) & P_{j} \\
\mathbf{0} & \mathbf{1} & \mathbf{1} & \mathbf{0} \\
\left(A_{i}-L_{i} C\right)^{T} P_{j} & \mathbf{1} & P_{i} & \mathbf{0} \\
P_{j} & \mathbf{0} & \mathbf{0} & \sigma_{e v} \mathbf{1}
\end{array}\right] .
$$

Hence, we have that for all $i, j \Psi_{i j} \succ \mathbf{0}$. For shortness we write $\hat{\xi}_{k}^{i}=\xi^{i}\left(\hat{\rho}_{k}\right)$ and $\xi_{k}^{i}=\xi^{i}\left(\rho_{k}\right)$. Multiplying $\Psi_{i j} \succ \mathbf{0}$ by $\hat{\xi}_{k}^{i}$ and summing, multiplying by $\hat{\xi}_{k+1}^{j}$ and summing, and using the Schur lemma yield

$$
\begin{aligned}
& {\left[\begin{array}{cc}
\mathcal{P}_{k} & \mathbf{0} \\
\mathbf{0} & \sigma_{e v} \mathbf{1}
\end{array}\right]-} \\
& {\left[\begin{array}{cc}
\mathcal{A}_{e}\left(\hat{\rho}_{k}\right)^{T} & \mathbf{1} \\
\mathbf{1} & \mathbf{0}
\end{array}\right]\left[\begin{array}{cc}
\mathcal{P}_{k+1} & \mathbf{0} \\
\mathbf{0} & \mathbf{1}
\end{array}\right]\left[\begin{array}{cc}
\mathcal{A}_{e}\left(\hat{\rho}_{k}\right) & \mathbf{1} \\
\mathbf{1} & \mathbf{0}
\end{array}\right] \succ \mathbf{0}}
\end{aligned}
$$

with $\mathcal{P}_{k}:=P\left(\hat{\xi}_{k}\right):=\sum_{i=1}^{N} \hat{\xi}_{k}^{i} P_{i}$ and $\mathcal{P}_{k+1}:=$ $P\left(\hat{\xi}_{k+1}\right)=\sum_{j=1}^{N} \hat{\xi}_{k+1}^{j} P_{j}$. Note that we used $\hat{\xi}_{k}, \hat{\xi}_{k+1} \in$ $\mathcal{S}$ (due to $\hat{\rho}_{k}, \hat{\rho}_{k+1} \in \Theta$ ). Hence, for all $e_{k} \in$ $\mathbb{R}^{n}$ and $v_{k} \in \mathbb{R}^{n}\left(\begin{array}{cc}e_{k}^{T} & v_{k}^{T}\end{array}\right) M\left(\begin{array}{l}e_{k} \\ v_{k}\end{array}\right) \geq 0$ with $M=\left[\begin{array}{cc}-\mathbf{1}+\mathcal{P}_{k}-\mathcal{A}_{e}\left(\hat{\rho}_{k}\right)^{T} \mathcal{P}_{k+1} \mathcal{A}_{e}\left(\hat{\rho}_{k}\right) & -\mathcal{A}_{e}\left(\hat{\rho}_{k}\right)^{T} \mathcal{P}_{k+1} \\ -\mathcal{P}_{k+1} \mathcal{A}_{e}\left(\hat{\rho}_{k}\right) & -\mathcal{P}_{k+1}+\sigma_{e v} \mathbf{1}\end{array}\right]$.

This implies for all $e_{k}$ and all $v_{k}$ that

$$
\begin{array}{r}
\left(\mathcal{A}_{e}\left(\hat{\rho}_{k}\right) e_{k}+v_{k}\right)^{T} \mathcal{P}_{k+1}\left(\mathcal{A}_{e}\left(\hat{\rho}_{k}\right) e_{k}+v_{k}\right)-e_{k}^{T} \mathcal{P}_{k} e_{k} \leq \\
-e_{k}^{T} e_{k}+\sigma_{e v} v_{k}^{T} v_{k} .
\end{array}
$$

This can be rewritten as (10a). We could base ourselves now on Theorem 1 to obtain ISS, but we proceed here to explicitly compute the ISS gain. From (10a) and (10b), one has

$$
V_{e}\left(e_{k+1}, \hat{\xi}_{k+1}\right) \leq\left(1-\frac{1}{\sigma_{e v}}\right) V_{e}\left(e_{k}, \hat{\xi}_{k}\right)+\sigma_{e v}\left\|v_{k}\right\|^{2}
$$

Applying (15) repetitively leads to

$$
\begin{aligned}
& V_{e}\left(e_{k}, \hat{\xi}_{k}\right) \leq \\
& \left(1-\frac{1}{\sigma_{e v}}\right)^{k} V_{e}\left(e_{0}, \hat{\xi}_{0}\right)+\sigma_{e v} \sum_{l=0}^{k-1}\left(1-\frac{1}{\sigma_{e v}}\right)^{k-l-1}\left\|v_{l}\right\|^{2} \\
& \quad \leq\left(1-\frac{1}{\sigma_{e v}}\right)^{k} V_{e}\left(e_{0}, \hat{\xi}_{0}\right)+\sigma_{e v}^{2}\|v\|_{\infty}^{2} .
\end{aligned}
$$

Finally, by using again (10b), taking the square root, we obtain the inequality

$$
\left\|e_{k}\right\| \leq \sqrt{\sigma_{e v}}\left(1-\frac{1}{\sigma_{e v}}\right)^{k / 2}\left\|e_{0}\right\|+\sigma_{e v}\|v\|_{\infty} .
$$

This inequality shows ISS with respect to $v$ with linear ISS gain $\gamma(s)=\sigma_{e v} s, s \in \mathbb{R}_{\geq 0}$.

In case the conditions of Theorem 2 hold, the polytopic observer (6) guarantees GES of the error dynamics (7) in the nominal case where $\rho_{k}=\hat{\rho}_{k}$ for all $k \in \mathbb{N}$ (as then $v_{k}=0, k \in \mathbb{N}$ ). In case $\rho_{k} \neq \hat{\rho}_{k}$, ISS (see (16)) guarantees only a steady state estimation error $e$ that is smaller than $\delta \sigma_{e v} \sup _{k \in \mathbb{N}}\left\|x_{k}\right\|$ with $\delta:=\sup \left\{\Delta A(\rho, \hat{\rho}) \mid\|\rho-\hat{\rho}\|_{\infty} \leq \Delta\right\}$. Hence, a kind of steady state relative error can be obtained in the sense that

$$
\frac{\limsup _{k \rightarrow \infty}\left\|e_{k}\right\|}{\lim \sup _{k \rightarrow \infty}\left\|x_{k}\right\|} \leq \delta \sigma_{e v}
$$

as was used also in [11] in the context of observer design for discontinuous PWA systems.

Remark 1: Note that the normalization of certain constants in (10) to 1 is without loss of generality as any ISS Lyapunov function $V_{e}$ for (7) can be multiplied by a sufficiently large positive constant to satisfy (10).

As mentioned, if the hypotheses of Theorem 2 are satisfied, the polytopic observer (6) guarantees GES of the error dynamics in the nominal case $\left(\rho_{k}=\hat{\rho}_{k}\right.$ for all $\left.k \in \mathbb{N}\right)$. Actually, the observer satisfies the matrix inequalities

$$
\begin{aligned}
\left(A_{i}-L_{i} C\right)^{T} \tilde{P}_{j}\left(A_{i}-L_{i} C\right)-\tilde{P}_{i} & \prec 0, i, j=1, \ldots, N \text { and } \\
\tilde{P}_{i} & \succ 0, i=1, \ldots, N, \quad \text { (17) }
\end{aligned}
$$

which are both necessary and sufficient conditions for the existence of a parameter-dependent quadratic Lyapunov function proving GES of the estimation error dynamics in the nominal case $\left(\hat{\rho}_{k}=\rho_{k}\right)$ [7,14]. Interestingly, the nominal conditions in (17) also guarantee that the hypotheses of Theorem 2 are satisfied (as will be shown in Theorem 3 below). This shows the non-conservatism of the LMIs (9) as the existence of a nominal observer for the exact LPV system, with a parameter-dependent quadratic Lyapunov function proving GES of the error dynamics, is sufficient for (9) to hold. This also shows that any GES observer for the exact LPV system has some degree of robustness.

Theorem 3: [9] If there exist $\tilde{P}_{i}$ and $L_{i}, i=1, \ldots, N$ such that (17) holds, then there are symmetric matrices $P_{i}$ and matrices $F_{i}, G_{i}, i=1, \ldots, N$ and a scalar $\sigma_{e v}$ satisfying for all $i, j=1, \ldots, N$ the LMIs (9). 


\section{STATE FeEdback DESIGN}

We now focus on the design of a state feedback for (4a) using an estimated state given by

$$
u_{k}=K\left(\hat{\rho}_{k}\right) \hat{x}_{k}=K\left(\hat{\rho}_{k}\right)\left(x_{k}-e_{k}\right)
$$

with $K\left(\hat{\rho}_{k}\right)=\sum_{i=1}^{N} \xi^{i}\left(\hat{\rho}_{k}\right) K_{i}$ and $e_{k}$ the estimation error. This results in the closed loop

$$
x_{k+1}=\mathcal{A}_{x}\left(\hat{\rho}_{k}\right) x_{k}+v_{k}-B K\left(\hat{\rho}_{k}\right) e_{k}
$$

with, as before, $v_{k}$ is given by (8) and

$$
\mathcal{A}_{x}\left(\hat{\rho}_{k}\right)=\sum_{i=1}^{N} \xi^{i}\left(\hat{\rho}_{k}\right) \underbrace{\left(A_{i}+B K_{i}\right)}_{A_{B K_{i}}}
$$

Again, we sometimes write $\hat{\xi}_{k}^{i}=\xi^{i}\left(\hat{\rho}_{k}\right)$ and $\xi_{k}^{i}=\xi^{i}\left(\rho_{k}\right)$. We now study ISS of (19).

Theorem 4: Assume that there exist symmetric matrices $Y_{i} \in \mathbb{R}^{n \times n}$, matrices $Z_{i} \in \mathbb{R}^{m \times n}, i=1, \ldots, N$ and scalars $\sigma_{x v}, \sigma_{x e}, \mu$ with $\mu>0$ and $\sigma_{x v} \geq 1$ satisfying for $i, j=$ $1, \ldots, N$ the LMI conditions

$$
\left[\begin{array}{ccccc}
Y_{i} & \mathbf{0} & \mathbf{0} & Y_{i} A_{i}^{T}+Z_{i}^{T} B^{T} & Y_{i} \\
\mathbf{0} & \sigma_{x v} \mathbf{1} & \mathbf{0} & \mathbf{1} & \mathbf{0} \\
\mathbf{0} & \mathbf{0} & \sigma_{x e} \mathbf{1} & -Z_{i}^{T} B^{T} & \mathbf{0} \\
A_{i} Y_{i}+B Z_{i} & \mathbf{1} & -B Z_{i} & Y_{j} & \mathbf{0} \\
Y_{i} & \mathbf{0} & \mathbf{0} & \mathbf{0} & \mathbf{1}
\end{array}\right] \succ \mathbf{0}
$$

and for $i=1, \ldots, N$

$$
Y_{i} \succeq \mu \mathbf{1},
$$

then the closed-loop system (19) with uncertainty set $\Theta$ for $\hat{\rho}$ and $K_{i}=Z_{i} Y_{i}^{-1}, i=1, \ldots, N$, is ISS with respect to $e$ and $v$ and $V_{x}\left(x_{k}, \hat{\xi}_{k}\right)=x_{k}^{T} \sum_{i=1}^{N} \hat{\xi}_{k}^{i} S_{i} x_{k}$ with $S_{i}=Y_{i}^{-1}$, $i=1, \ldots, N$, is an ISS Lyapunov function that satisfies for all $\hat{\xi}_{k}, \hat{\xi}_{k+1} \in \mathcal{S}$, all $x_{k} \in \mathbb{R}^{n}$, all $e_{k} \in \mathbb{R}^{n}$ and all $v_{k} \in \mathbb{R}^{n}$

$$
\begin{aligned}
& V_{x}\left(x_{k+1}, \hat{\xi}_{k+1}\right)-V_{x}\left(x_{k}, \hat{\xi}_{k}\right) \leq \\
& \quad-\left\|x_{k}\right\|^{2}+\sigma_{x v}\left\|v_{k}\right\|^{2}+\mu^{-2} \sigma_{x e}\left\|e_{k}\right\|^{2}
\end{aligned}
$$

and

$$
\left\|x_{k}\right\|^{2} \leq V_{x}\left(x_{k}, \hat{\xi}_{k}\right) \leq \sigma_{x v}\left\|x_{k}\right\|^{2} .
$$

Proof: Assume that the LMIs in (20) are feasible and define $S_{i}:=Y_{i}^{-1}$. Premultiply the LMIs in (20a) by $\mathcal{T}=$ $\operatorname{diag}\left(S_{i}, \mathbf{1}, S_{i}, \mathbf{1}, \mathbf{1}\right)$, postmultiply it by $\mathcal{T}^{T}=\mathcal{T}$ and apply the Schur lemma to arrive for $i, j=1, \ldots, N$ at

$$
\left[\begin{array}{cccc}
S_{i}-\mathbf{1} & \mathbf{0} & \mathbf{0} & \left(A_{i}+B K_{i}\right)^{T} \\
\mathbf{0} & \sigma_{x v} \mathbf{1} & \mathbf{0} & \mathbf{1} \\
\mathbf{0} & \mathbf{0} & \sigma_{x e} S_{i}^{2} & -K_{i}^{T} B^{T} \\
A_{i}+B K_{i} & \mathbf{1} & -B K_{i} & S_{j}^{-1}
\end{array}\right] \succ \mathbf{0} .
$$

Multiply (22) by $\hat{\xi}_{k}^{i}$, sum for $i=1, \ldots, N$ (note that $\sum_{i=1}^{N} \hat{\xi}_{k}^{i}=1$, since $\hat{\rho}_{k} \in \Theta$ ) and use the Schur lemma pivoting again around the south east block to obtain for $j=1, \ldots, N$

$$
\left[\begin{array}{ccc}
T_{11} & (\bullet)^{T} & (\bullet)^{T} \\
-S_{j} \sum_{i=1}^{N} \hat{\xi}_{k}^{i} A_{B K i} & -S_{j}+\sigma_{x v} \mathbf{1} & (\bullet)^{T} \\
T_{13} & \left(\sum_{i=1}^{N} \hat{\xi}_{k}^{i} B K_{i}\right)^{T} S_{j} & T_{33}
\end{array}\right] \succ \mathbf{0}
$$

where

$T_{11}=-\left(\sum_{i=1}^{N} \hat{\xi}_{k}^{i} A_{B K i}\right)^{T} S_{j}\left(\sum_{i=1}^{N} \hat{\xi}_{k}^{i} A_{B K i}\right)+\sum_{i=1}^{N} \hat{\xi}_{k}^{i} S_{i}-\mathbf{1}$,
$T_{13}=\left(\sum_{i=1}^{N} \hat{\xi}_{k}^{i} B K_{i}\right)^{T} S_{j}\left(\sum_{i=1}^{N} \hat{\xi}_{k}^{i} A_{B K_{i}}\right)$, and

$T_{33}=-\left(\sum_{i=1}^{N} \hat{\xi}_{k}^{i} B K_{i}\right)^{T} S_{j}\left(\sum_{i=1}^{N} \hat{\xi}_{k}^{i} B K_{i}\right)+\sigma_{x e} \sum_{i=1}^{N} \hat{\xi}_{k}^{i} S_{i}^{2}$. Using that $S_{i}^{2} \preceq \mu^{-2} \mathbf{1}, i=1, \ldots, N$ due to the second LMIs in (20), multiplying (23) by $\hat{\xi}_{k+1}^{j}$ and summing for $j=1, \ldots, N$ (note that $\sum_{j=1}^{N} \hat{\xi}_{k+1}^{j}=1$, since $\hat{\rho}_{k+1} \in \Theta$ ) leads (in a similar way as we obtained (10a) in the proof of Theorem 2) to

$$
\begin{aligned}
& V_{x}\left(x_{k+1}, \hat{\xi}_{k+1}\right)-V_{x}\left(x_{k}, \hat{\xi}_{k}\right) \leq \\
& \quad-\left\|x_{k}\right\|^{2}+\sigma_{x v}\left\|v_{k}\right\|^{2}+\mu^{-2} \sigma_{x e}\left\|e_{k}\right\|^{2} .
\end{aligned}
$$

As (22) implies $S_{i} \succ \mathbf{1}$ and $S_{i} \preceq \sigma_{x v} 1$, we have for all $x_{k} \in \mathbb{R}^{n}$ and $\xi_{k} \in \mathcal{S}$ that (21b) holds. From Theorem 1 it follows now that the closed-loop system is ISS with respect to $v$ and $e$.

The following corollary applies when the full state $x_{k}$ is known (i.e. $e_{k}=0$ for all $k \in \mathbb{N}$ ).

Corollary 1: Let the hypotheses of Theorem 4 be satisfied. Then the LPV system consisting of (4a) and the state feedback $u_{k}=K\left(\hat{\rho}_{k}\right) x_{k}$ with uncertainty set $\Theta$ for $\rho$ and $K_{i}=$ $Z_{i} Y_{i}^{-1}, i=1, \ldots, N$ is GES for all uncertainties satisfying $\|\Delta A(\rho, \hat{\rho})\| \leq \delta$, when $\delta<\frac{1}{\sigma_{r v}}$.

Proof: From (21a) with $e_{k}=0, k \in \mathbb{N}$ and $v_{k}=$ $\Delta A\left(\rho_{k}, \hat{\rho}_{k}\right) x_{k}$ it follows that

$$
V_{x}\left(x_{k+1}, \hat{\xi}_{k+1}\right)-V_{x}\left(x_{k}, \hat{\xi}_{k}\right) \leq-\left(1-\sigma_{x v} \delta\right)\left\|x_{k}\right\|^{2} .
$$

Together with (21b) this proves GES on the basis of Theorem 1.

An analogous result to Theorem 3 can also be shown for the state feedback design. In particular, a nominal state feedback $u_{k}=K\left(\rho_{k}\right) x_{k}$ with $K\left(\rho_{k}\right)=\sum_{i=1}^{N} \xi^{i}\left(\rho_{k}\right) K_{i}$ (i.e. without estimation error $\left(e_{k}=0, k \in \mathbb{N}\right)$ and exact knowledge of the parameters, $\rho_{k}=\hat{\rho}_{k}, k \in \mathbb{N}$ ) coupled to the LPV system (4a) is GES if there are $K_{i}, \tilde{S}_{i}, i=1, \ldots, N$ such that

$$
\begin{aligned}
\left(A_{i}+B K_{i}\right)^{T} \tilde{S}_{j}\left(A_{i}+B K_{i}\right)-\tilde{S}_{i} & \prec \mathbf{0}, i, j=1, \ldots, N \text { and } \\
\tilde{S}_{i} & \succ \mathbf{0}, i=1, \ldots, N . \quad \text { (26) }
\end{aligned}
$$

Clearly, a state feedback (18) that renders (19) ISS (proved by parameter-dependent quadratic ISS Lyapunov functions) certainly satisfies (26). Interestingly, the converse also holds in the sense that a nominally stabilizing state feedback for (4a) satisfying (26) has some robustness properties in the sense that (21) holds for some $V_{x}$ and even stronger, the LMIs in (20) are feasible. This clearly indicates the non-conservatism of the derived LMIs in Theorem 4. However, note that (26) does not allow any minimization of the ISS gains, while the results of Theorem 4 do.

Theorem 5: [9] Suppose that there exist $K_{i}, \tilde{S}_{i}, i=$ $1, \ldots, N$ such that (26) is satisfied. Then there are symmetric matrices $Y_{i}$ and matrices $Z_{i}, i=1, \ldots, N$ and scalars $\sigma_{x v}, \sigma_{x e}, \mu$ with $\mu>0$ and $\sigma_{x v} \geq 1$ satisfying the LMIs (20) for $i, j=1, \ldots, N$.

\section{OBSERVER-BASED CONTROL DESIGN}

Next we will show that the separate design of the observer as in section IV and a state feedback as in section V leads to a stabilizing output-based controller for some nontrivial level 
of uncertainty $\delta:=\sup \left\{\|\Delta A(\rho, \hat{\rho})\| \mid\|\rho-\hat{\rho}\|_{\infty} \leq \Delta\right\}$. The closed-loop system is given by

$$
\left(\begin{array}{c}
x_{k+1} \\
e_{k+1}
\end{array}\right)=\left[\begin{array}{cc}
A\left(\rho_{k}\right)+B K\left(\hat{\rho}_{k}\right) & -B K\left(\hat{\rho}_{k}\right) \\
A\left(\rho_{k}\right)-A\left(\hat{\rho}_{k}\right) & A\left(\hat{\rho}_{k}\right)-L\left(\hat{\rho}_{k}\right) C
\end{array}\right]\left(\begin{array}{c}
x_{k} \\
e_{k}
\end{array}\right) .
$$

Theorem 6: Let an observer (6) that satisfies the hypotheses of Theorem 2 and a state feedback law that satisfies the hypotheses of Theorem 4 be given. Then for any $\max \left\{1-\frac{1}{\sigma_{e v}}, 1-\frac{1}{\sigma_{x v}}\right\} \leq \varepsilon<1$ and any $0<\beta \leq \frac{1-(1-\varepsilon) \sigma_{e v}}{\mu^{-2} \sigma_{x e}}$ the closed-loop system (27) is GES with decay factor equal to $\sqrt{\varepsilon}$ for all uncertainties satisfying

$$
\|\Delta A(\rho, \hat{\rho})\| \leq \delta:=\sqrt{\frac{\beta\left(1-(1-\varepsilon) \sigma_{x v}\right)}{\sigma_{e v}+\beta \sigma_{x v}}} .
$$

Proof: Consider the candidate Lyapunov function $V_{\beta}\left(x_{k}, e_{k}, \hat{\xi}_{k}\right):=\beta V_{x}\left(x_{k}, \hat{\xi}_{k}\right)+V_{e}\left(e_{k}, \hat{\xi}_{k}\right)$ for the closed-loop system (27) with $\beta>0$. From (10) and (21) and noting that $v_{k}=\Delta A\left(\rho_{k}, \hat{\rho}_{k}\right) x_{k}$ with

$$
\delta=\sup \left\{\|\Delta A(\rho, \hat{\rho})\| \mid\|\rho-\hat{\rho}\|_{\infty} \leq \Delta\right\}
$$

we have that

$$
\begin{aligned}
& \Delta V_{\beta}\left(x_{k}, e_{k}, \hat{\xi}_{k}, \hat{\xi}_{k+1}\right) \leq \\
& \quad\left(-\beta+\beta \sigma_{x v} \delta^{2}+\sigma_{e v} \delta^{2}\right)\left\|x_{k}\right\|^{2}-\left(1-\beta \mu^{-2} \sigma_{x e}\right)\left\|e_{k}\right\|^{2},
\end{aligned}
$$

where $\quad \Delta V_{\beta}\left(x_{k}, e_{k}, \hat{\xi}_{k}, \hat{\xi}_{k+1}\right):=V_{\beta}\left(x_{k+1}, e_{k+1}, \hat{\xi}_{k+1}\right)-$ $V_{\beta}\left(x_{k}, e_{k}, \hat{\xi}_{k}\right)$ with $\left(x_{k+1}^{T}, e_{k+1}^{T}\right)^{T}$ as in (27). To obtain GES with decay factor $\sqrt{\varepsilon}$ it suffices to guarantee $V_{\beta}\left(x_{k+1}, e_{k+1}, \hat{\xi}_{k+1}\right) \leq \varepsilon V_{\beta}\left(x_{k}, e_{k}, \hat{\xi}_{k}\right)$ as $V_{\beta}$ can be bounded by quadratic $\mathcal{K}$ functions $\alpha_{1}(s)=a s^{2}$ and $\alpha_{2}(s)=b s^{2}$ as in (3a) in the norm $\left\|\left(x_{k}^{T}, e_{k}^{T}\right)^{T}\right\|$. To obtain this inequality it is sufficient to have

$$
\Delta V_{\beta}\left(x_{k}, e_{k}, \hat{\xi}_{k}, \hat{\xi}_{k+1}\right) \leq-(1-\varepsilon)\left(\beta \sigma_{x v}\left\|x_{k}\right\|^{2}+\sigma_{e v}\left\|e_{k}\right\|^{2}\right),
$$

because $V_{\beta}\left(x_{k}, e_{k}, \hat{\xi}_{k}\right) \leq \beta \sigma_{x v}\left\|x_{k}\right\|^{2}+\sigma_{e v}\left\|e_{k}\right\|^{2}$. Due to (28), the inequality (29) holds when (i) $\beta-\beta \sigma_{x v} \delta^{2}-\sigma_{e v} \delta^{2} \geq$ $(1-\varepsilon) \beta \sigma_{x v}$ and (ii) $1-\beta \mu^{-2} \sigma_{x e} \geq(1-\varepsilon) \sigma_{e v}$. Obviously, under the hypotheses of the theorem these conditions are true, which completes the proof.

It is of interest to find the Lyapunov function $V_{\beta}$ that provides the largest robustness in terms of $\delta$. To maximize the value for $\delta^{2}$ (for a fixed value of the decay factor $\sqrt{\varepsilon}$ ) it is clear that we have to maximize $f(\beta):=\frac{\beta}{\sigma_{e v}+\beta \sigma_{x v}}$. Since $\frac{d f(\beta)}{d \beta}=\frac{\sigma_{e v}}{\left(\sigma_{e v}+\beta \sigma_{x v}\right)^{2}} \geq 0$, the maximum is obtained for the largest allowable value of $\beta$, which is $\frac{1-(1-\varepsilon) \sigma_{e v}}{\mu^{-2} \sigma_{x e}}$ and thus the maximum of $\delta$ is

$$
\delta(\varepsilon)=\sqrt{\frac{\left(1-[1-\varepsilon] \sigma_{e v}\right)\left(1-[1-\varepsilon] \sigma_{x v}\right)}{\mu^{-2} \sigma_{x e} \sigma_{e v}+\left(1-[1-\varepsilon] \sigma_{e v}\right) \sigma_{x v}}} .
$$

Hence, we obtained the following corollary.

Corollary 2: Let an observer (6) that satisfies the hypotheses of Theorem 2 and a state feedback law that satisfies the hypotheses of Theorem 4 be given. Then for any $\max \left\{1-\frac{1}{\sigma_{e v}}, 1-\frac{1}{\sigma_{x v}}\right\} \leq \varepsilon<1$ the closed-loop system (27) is GES with decay factor equal to $\sqrt{\varepsilon}$ for all uncertainties satisfying $\|\Delta A(\rho, \hat{\rho})\| \leq \delta(\varepsilon)$ with $\delta(\varepsilon)$ as in (30).

Suppose we now would like to find the value of $\varepsilon$ such that the admissible uncertainty level $\delta(\varepsilon)$ is maximal. Since it can be inspected that $\frac{d \delta^{2}(\varepsilon)}{d \varepsilon}>0$ for any $\max \left\{1-\frac{1}{\sigma_{e v}}, 1-\frac{1}{\sigma_{x v}}\right\} \leq$ $\varepsilon<1$, maximizing robustness requires maximizing (actually taking supremum of) $\varepsilon$ and thus taking it close to 1 . This yields that the maximal value of $\delta$ can become arbitrarily close to

$$
\delta(1)=\sqrt{\frac{1}{\mu^{-2} \sigma_{x e} \sigma_{e v}+\sigma_{x v}}},
$$

while still guaranteeing stability. Hence, for maximizing robustness in terms of maximizing $\delta(\varepsilon)$, we should maximize $\varepsilon$ meaning that the performance in terms of the decay factor $\sqrt{\varepsilon}$ is worst. As such, we encountered a "classical" tradeoff between robustness and performance.

The reasoning above maximizes robustness for fixed values of $\sigma_{x v}, \sigma_{e v}$ and $\sigma_{x e}$. Since we have determined the maximum $\delta(1)$ as in (31) given these $\sigma$ 's, we can now optimize robustness by appropriately selecting the gains $L_{i}$ and $K_{i}$, $i=1, \ldots, N$. From (31) it is clear that we have to minimize $\mu^{-2} \sigma_{x e} \sigma_{e v}+\sigma_{x v}$ to get the maximal value for the uncertainty level (just below) $\delta(1)=\sqrt{\frac{1}{\mu^{-2} \sigma_{x e} \sigma_{e v}+\sigma_{x v}}}$, while still guaranteeing GES (for decay factor just below 1). This gives rise to the following procedure to get maximal robustness in the mismatch between the scheduling parameter $\hat{\rho}_{k}$ and the actual one $\rho_{k}$ as reflected in $\delta$, while still guaranteeing GES.

\section{Design procedure}

Step 1: Minimize $\sigma_{e v}$ subject to (9) for $i, j=1, \ldots, N$. This gives the minimum $\sigma_{e v}^{*}$ and the corresponding observer gains $L_{i}, i=1, \ldots, N$.

Step 2: Given $\sigma_{e v}^{*}$ as in Step 1. Fix $\mu>0$ and minimize the expression $\mu^{-2} \sigma_{x e} \sigma_{e v}^{*}+\sigma_{x v}$ subject to the LMIs given in (20). This results in the feedback gains $K_{i}$, $i=1, \ldots, N$.

The optimization problems in Step 1 and 2 are convex problems as we are minimizing linear costs subject to LMI constraints. Step 2 might even be extended by performing a line search in $\mu$ and applying the above procedure repetitively. Once, the minimal value $\mu^{*-2} \sigma_{x e}^{*} \sigma_{e v}^{*}+\sigma_{x v}^{*}$ is found, one can on the basis of Theorem 6 and (30) still make tradeoffs between transient performance in terms of the decay factor $\sqrt{\varepsilon}$ and robustness in terms of $\delta(\varepsilon)$. Letting $\varepsilon$ increase from $\max \left\{1-\frac{1}{\sigma_{e v}}, 1-\frac{1}{\sigma_{x v}}\right\}$ (maximal performance, minimal robustness) to 1 (minimal performance, maximal robustness), tradeoff curves between performance and robustness are obtained as was already indicated in Corollary 2 .

\section{ILLUSTRATIVE EXAMPLE}

Consider the LPV system (4) with

$$
\begin{gathered}
A\left(\rho_{k}\right)=\left[\begin{array}{ccc}
0.25 & 1 & 0 \\
0 & 0.1 & 0 \\
0 & 0 & 0.6+\rho_{k}
\end{array}\right], \quad B=\left[\begin{array}{l}
1 \\
0 \\
1
\end{array}\right], \\
C=\left[\begin{array}{ll}
1 & 0
\end{array}\right], \quad D=0
\end{gathered}
$$




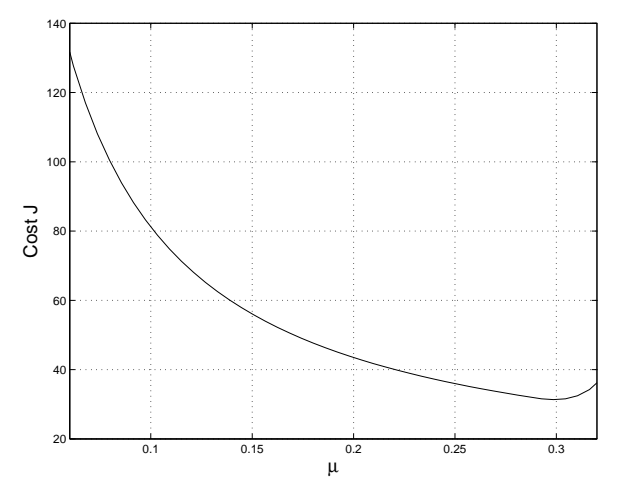

Fig. 1. Line search $\mu$

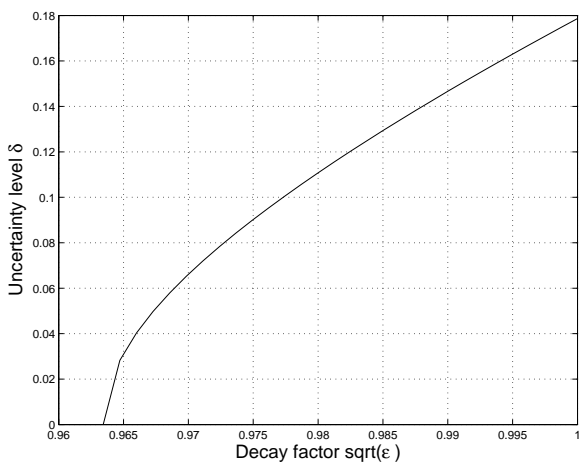

Fig. 2. Tradeoff performance/robustness

and $\rho_{k} \in[0,0.5], k \in \mathbb{N}$. In this case we can take the functions $\xi^{1}(\rho)=\frac{0.5-\rho}{0.5}$ and $\xi^{2}(\rho)=\frac{\rho}{0.5}$ with $A_{1}=$ $A(0)$ and $A_{2}=A(0.5)$. The observer is designed using Theorem 2 along with the optimization problem in Step 1. The optimal solution is given by $\sigma_{e v}^{*}=5.8277$ with observer gains $L_{1}=\left[\begin{array}{lll}-0.0835 & -0.0011 & 0.3870\end{array}\right]^{T}$ and $L_{2}=\left[\begin{array}{lll}-0.0835 & -0.0011 & 0.7094\end{array}\right]^{T}$. With this optimal observer and the associated slope of the linear ISS gain $\sigma_{e v}^{*}$, a line search involving $\mu>0$ is performed in order to minimize the cost $J=\mu^{-2} \sigma_{x e} \sigma_{e v}^{*}+\sigma_{x v}$ subject to the LMIs given in (20) for all $i, j$ (Step 2). Fig. 1 shows the minimum of $J$ for each fixed $\mu$, which is the smallest for $\mu^{*}=0.2986$ yielding $\sigma_{x e}^{*}=0.2663$ and $\sigma_{x v}^{*}=13.9284$ and corresponds to the controller gains $K_{1}=\left[\begin{array}{lll}-0.0327 & -0.1241-0.2387\end{array}\right]$, $K_{2}=[0.0005-0.0010-0.6148]$.

As a consequence, the maximum level of uncertainty is

$$
\delta_{\text {max }}^{*}=\sqrt{\frac{1}{\mu^{*-2} \sigma_{x e}^{*} \sigma_{e v}^{*}+\sigma_{x v}^{*}}}=0.1786 .
$$

Hence, for $\Delta A\left(\rho_{k}, \hat{\rho}_{k}\right)=\left|\rho_{k}-\hat{\rho}_{k}\right| \leq \delta<0.1786$ GES of the closed-loop system (27) is guaranteed (with a decay factor close to 1$)$. Letting $\varepsilon$ increase from $\max \left\{1-\frac{1}{\sigma_{e v}^{*}}, 1-\frac{1}{\sigma_{x v}^{*}}\right\}$ to 1 leads to the tradeoff curves between performance in terms of the decay factor $\sqrt{\varepsilon}$ and robustness to uncertainty $\Delta A\left(\rho_{k}, \hat{\rho}_{k}\right)$ in terms of $\delta$ as depicted in Fig. 2.

\section{CONCLUSIONS}

In this paper the design of robustly stabilizing outputbased feedback controllers is considered for discrete-time LPV systems in which the scheduling parameters are only known up to a given precision. The output-based controllers are obtained using a separate design of the observer and the state feedback and we showed that the interconnection of the LPV plant, observer and state feedback leads to a globally exponentially stable closed-loop system for certain levels of mismatch between estimated and true parameters. The nonconservatism of our approach is demonstrated by showing that well known conditions for nominally stabilizing observers and feedbacks (i.e. without mismatch between true and available parameters) imply our LMI-based conditions. The flexibility in the framework allows to construct the controller that guarantees global exponential stability for the largest level of parameter uncertainty and to make tradeoffs between transient performance in terms of decay factors and robustness with respect to parameter uncertainty.

\section{REFERENCES}

[1] A. Alessandri, M. Baglietto, and G. Battistelli. Luenberger observers for switching discrete-time linear systems. Int. J. Control, 80(12):19311943, 2007.

[2] P. Apkarian and P. Gahinet. A convex characterization of gain-scheduled Hinfinity controllers. IEEE Trans. on Automatic Control, 40:853-864, 1995.

[3] G. Becker and A. Packard. Robust performance of linear parametrically varying systems using parametrically-dependent linear feedback. Syst. Contr. Lett., 23:205-215, 1994.

[4] F. Blanchini and S. Miani. Stabilization of LPV systems: state feedback, state estimation, and duality. SIAM J. Control Optim., 42:76-97, 2003.

[5] F. Blanchini, S. Miani, and F. Mesquine. A separation principle for linear switching systems and parametrization of all stabilizing controllers. IEEE Transactions on Automatic Control, 54(2):279-291, 2009.

[6] G. Chesi, A. Garulli, A. Tesi, and A. Vicino. Homogeneous Polynomial Forms for Robustness Analysis of Uncertain Systems. Springer, 2009.

[7] J. Daafouz and J. Bernussou. Parameter dependent Lyapunov functions for discrete time systems with time varying parametric uncertainties. Systems and Control Letters, 43:355-359, 2001.

[8] J. Daafouz, J. Bernussou, and J.C. Geromel. On inexact LPV control design of continuous-time polytopic systems. IEEE Trans. Automatic Control, 53:1674-1678, 2008.

[9] W.P.M.H. Heemels, J. Daafouz, and G. Millerioux. Observer-based control of discrete-time LPV systems with uncertain parameters. Technical Report DCT 2009.121, Eindhoven University of Technology, dept. Mechanical Eng., Dynamics and Control Technology group, 2009.

[10] Z.-P. Jiang and Y. Wang. Input-to-state stability for discrete-time nonlinear systems. Automatica, 37:857-869, 2001.

[11] A.Lj. Juloski, W.P.M.H. Heemels, and S. Weiland. Observer design for a class of piecewise linear systems. Intern. J. Robust and Nonlinear Control, 17(15):1387-1404, 2007.

[12] I.E. Kose and F. Jabbari. Control of LPV systems with partly-measured parameters. Proceedings of the 36th IEEE Conference on Decision and Control, 1997.

[13] M. Lazar, D. Munoz de la Pena, W.P.M.H. Heemels, and T. Alamo. On the stability of min-max nonlinear model predictive control. Syst. Contr. Lett., 57(1):39-48, 2008.

[14] G. Millerioux, L. Rosier, G. Bloch, and J. Daafouz. Bounded state reconstruction error for LPV systems with estimated parameters. IEEE Trans. Automatic Control, pages 1385-1389, 2004.

[15] W.J. Rugh and J.S. Shamma. A survey of research on gain-scheduling. Automatica, 36:1401-1425, 2000.

[16] C. Scherer. LPV control and full block multipliers. Automatica, 37:361$375,2001$.

[17] J. Shamma and M. Athans. Guaranteed properties of gain scheduled control of linear parameter-varying plants. Automatica, 27:559-564, 1991.

[18] E. D. Sontag. Smooth stabilization implies coprime factorization. IEEE Trans. Automatic Control, 34:435-443, 1989.

[19] N. van de Wouw and A. Pavlov. Tracking and synchronisation for a class of PWA systems. Automatica, 44(11):2909-2915, 2008. 